\title{
FIXED POINTS SUBGROUPS BY TWO INVOLUTIVE AUTOMORPHISMS $\sigma, \gamma$ OF COMPACT EXCEPTIONAL LIE GROUPS $F_{4}, E_{6}$ AND $E_{7}$
}

\author{
By \\ Toshikazu MiYashita
}

\section{Introduction}

For simply connected compact exceptional Lie groups $G=F_{4}, E_{6}$ and $E_{7}$, we consider two involutions $\sigma, \gamma$ and determine the group structure of subgroups $G^{\sigma, \gamma}$ of $G$ which are the intersection $G^{\sigma} \cap G^{\gamma}$ of the fixed points subgroups of $G^{\sigma}$ and $G^{\gamma}$. The motivation is as follows. In [1], we determine the group structure of $\left(F_{4}\right)^{\sigma, \sigma^{\prime}},\left(E_{6}\right)^{\sigma, \sigma^{\prime}}$ and $\left(E_{7}\right)^{\sigma, \sigma^{\prime}}$, and in [2], we also determine the group structure of $\left(G_{2}\right)^{\gamma, \gamma^{\prime}},\left(F_{4}\right)^{\gamma, \gamma^{\prime}}$ and $\left(E_{6}\right)^{\gamma, \gamma^{\prime}}$. So, in this paper, we try to determine the type of groups $\left(F_{4}\right)^{\sigma, \gamma},\left(E_{6}\right)^{\sigma, \gamma}$ and $\left(E_{7}\right)^{\sigma, \gamma}$. Our results are the following second columns. The first columns are already known in [3], [4] or [5] and these play an important role to obtain our results. In Table 1 , the results of the group structure of $G^{\sigma, \gamma}$ are obtained by the result of $G^{\gamma}$ and in Table 2, ones are obtained by the result of $G^{\sigma}$. In this paper, we show the proof of the results of the first and the second line of Table 1 and the third line of Table 2 .

\section{Acknowledgment}

The author is grateful to Professor Ichiro Yokota for his valuable comments. As for the group $\left(E_{8}\right)^{\sigma, \gamma}$, we can not realize explicitly, however we conjecture

$$
\left(E_{8}\right)^{\sigma, \gamma} \cong(\operatorname{Spin}(4) \times \operatorname{Spin}(12)) /\left(Z_{2} \times Z_{2}\right) .
$$

REMARK. In $E_{7}$, since $\gamma$ is conjugate to $-\sigma$, we have $\left(E_{7}\right)^{\gamma} \cong\left(E_{7}\right)^{\sigma}$. (In detail, see [4].) Note that the results of Table 1 and Table 2 are the same as a set, however they are different as realizations. 
Table 1

\begin{tabular}{lll}
\hline$G$ & \multicolumn{1}{c}{$G^{\gamma}$} & \multicolumn{1}{c}{$G^{\sigma, \gamma}$} \\
\hline$F_{4}$ & $(S p(1) \times S p(3)) / Z_{2}$ & $(S p(1) \times S p(1) \times S p(2)) / Z_{2}$ \\
$E_{6}$ & $(S p(1) \times S U(6)) / Z_{2}$ & $(S p(1) \times S(U(2) \times U(4))) / Z_{2}$ \\
$E_{7}$ & $(S U(2) \times S p i n(12)) / Z_{2}$ & $(S U(2) \times S p i n(4) \times \operatorname{Spin}(8)) /\left(Z_{2} \times Z_{2}\right)$ \\
\hline
\end{tabular}

Table 2

\begin{tabular}{lll}
\hline$G$ & \multicolumn{1}{c}{$G^{\sigma}$} & \multicolumn{1}{c}{$G^{\sigma, \gamma}$} \\
\hline$F_{4}$ & $\operatorname{Spin}(9)$ & $(\operatorname{Spin}(4) \times \operatorname{Spin}(5)) / Z_{2}$ \\
$E_{6}$ & $(U(1) \times \operatorname{Spin}(10)) / Z_{4}$ & $(U(1) \times \operatorname{Spin}(4) \times \operatorname{Spin}(6)) / Z_{2}$ \\
$E_{7}$ & $(S U(2) \times \operatorname{Spin}(12)) / Z_{2}$ & $(S U(2) \times \operatorname{Spin}(4) \times \operatorname{Spin}(8)) /\left(Z_{2} \times Z_{2}\right)$ \\
\hline
\end{tabular}

\section{Notation}

(1) For a group $G$ and an element $s$ of $G$, we denote $\{g \in G \mid s g=g s\}$ by $G^{s}$.

(2) For a transformation group $G$ of a space $M$, the isotropy subgroup of $G$ at $m_{1}, \ldots, m_{k} \in M$ is denoted by $G_{m_{1}, \ldots, m_{k}}=\left\{g \in G \mid g m_{1}=m_{1}, \ldots, g m_{k}=m_{k}\right\}$.

(3) For a $\boldsymbol{R}$-vector space $V$, its complexification $\{u+i v \mid u, v \in V\}$ is denoted by $V^{C}$. The complex conjugation in $V^{C}$ is denoted by $\tau: \tau(u+i v)=u-i v$. In particular, the complexification of $\boldsymbol{R}$ is briefly denoted by $C: \boldsymbol{R}^{C}=C$.

(4) For a Lie group $G$, the Lie algebra of $G$ is denoted by the corresponding German small letter $\mathfrak{g}$. For example, $\mathfrak{s o}(n)$ is the Lie algebra of the group $S O(n)$.

(5) Although we will give all definitions used in the following Sections, if in case of insufficiency, refer to [3], [4] or [5].

\section{Group $F_{4}$}

We use the same notation as in [1], [2] or [5] (however, some will be rewritten). For example, the Cayley algebra $\mathfrak{C}=\boldsymbol{H} \oplus \boldsymbol{H} e_{4}$, the exceptional Jordan algebra $\mathfrak{I}=\left\{X \in M(3, \mathfrak{C}) \mid X^{*}=X\right\}$, the Jordan multiplication $X \circ Y$, the inner product $(X, Y)$ and the elements $E_{1}, E_{2}, E_{3} \in \mathfrak{I}$, the group $F_{4}=\left\{\alpha \in \operatorname{Iso}_{R}(\mathfrak{J}) \mid \alpha(X \circ Y)=\right.$ $\alpha X \circ \alpha Y\}$.

We define $\boldsymbol{R}$-linear transformations $\sigma$ and $\gamma$ of $\mathfrak{I}$ by

$$
\sigma X=\sigma\left(\begin{array}{ccc}
\xi_{1} & x_{3} & \bar{x}_{2} \\
\bar{x}_{3} & \xi_{2} & x_{1} \\
x_{2} & \bar{x}_{1} & \xi_{3}
\end{array}\right)=\left(\begin{array}{ccc}
\xi_{1} & -x_{3} & -\bar{x}_{2} \\
-\bar{x}_{3} & \xi_{2} & x_{1} \\
-x_{2} & \bar{x}_{1} & \xi_{3}
\end{array}\right), \quad \gamma X=\left(\begin{array}{ccc}
\xi_{1} & \gamma x_{3} & \overline{\gamma x}_{2} \\
\overline{\gamma x_{3}} & \xi_{2} & \gamma x_{1} \\
\gamma x_{2} & \overline{\gamma x}_{1} & \xi_{3}
\end{array}\right),
$$


respectively, where $\gamma x_{k}=\gamma\left(m_{k}+a_{k} e_{4}\right)=m_{k}-a_{k} e_{4}, x_{k}=m_{k}+a_{k} e_{4} \in \boldsymbol{H} \oplus \boldsymbol{H} e_{4}=\mathfrak{C}$. Then, $\sigma, \gamma \in F_{4}$ and $\sigma^{2}=\gamma^{2}=1 . \sigma$ and $\gamma$ are commutative. From $\sigma \gamma=\gamma \sigma$, we have

$$
\left(F_{4}\right)^{\sigma} \cap\left(F_{4}\right)^{\gamma}=\left(\left(F_{4}\right)^{\sigma}\right)^{\gamma}=\left(\left(F_{4}\right)^{\gamma}\right)^{\sigma} .
$$

Hence, this group will be denoted briefly by $\left(F_{4}\right)^{\sigma, \gamma}$.

Proposition 1.1. $\left(F_{4}\right)^{\gamma} \cong(S p(1) \times S p(3)) / Z_{2}, Z_{2}=\{(1, E),(-1,-E)\}$.

Proof. The isomorphism is induced by the homomorphism $\varphi: S p(1) \times S p(3) \rightarrow$ $\left(F_{4}\right)^{\gamma}, \varphi(p, A)(M+\boldsymbol{a})=A M A^{*}+p \boldsymbol{a} A^{*}, M+\boldsymbol{a} \in \mathfrak{I}(3, \boldsymbol{H}) \oplus \boldsymbol{H}^{3}=\mathfrak{I} . \quad$ (In detail, see [3], [5].)

Lemma 1.2. $\varphi: S p(1) \times S p(3) \rightarrow\left(F_{4}\right)^{\gamma}$ of Proposition 1.1 satisfies $\sigma \varphi(p, A) \sigma=$ $\varphi\left(p, I_{1} A I_{1}\right)$, where $I_{1}=\operatorname{diag}(-1,1,1)$.

Proof. From $\sigma=\varphi\left(-1, I_{1}\right)$, we have the required one.

Now, we shall determine the group structure of $\left(F_{4}\right)^{\sigma, \gamma}=\left(\left(F_{4}\right)^{\gamma}\right)^{\sigma}=\left(\left(F_{4}\right)^{\sigma}\right)^{\gamma}=$ $\left(F_{4}\right)^{\sigma} \cap\left(F_{4}\right)^{\gamma}$.

THEOREM 1.3. $\left(F_{4}\right)^{\sigma, \gamma} \cong(S p(1) \times S p(1) \times S p(2)) / Z_{2}, Z_{2}=\{(1,1, E),(-1,-1$, $-E)\}$.

Proof. We define a map $\varphi_{4}: S p(1) \times S p(1) \times S p(2) \rightarrow\left(F_{4}\right)^{\sigma, \gamma}$ by

$$
\varphi_{4}(p, q, B)(M+a)=\left(\begin{array}{c|cc}
q & 0 & 0 \\
\hline 0 & B \\
0 & B
\end{array}\right) M\left(\begin{array}{c|cc}
q & 0 & 0 \\
\hline 0 & B \\
0 & B
\end{array}\right)^{*}+p a\left(\begin{array}{c|cc}
q & 0 & 0 \\
\hline 0 & B \\
0 & B
\end{array}\right)^{*}
$$

$M+a \in \mathfrak{J}(3, \boldsymbol{H}) \oplus \boldsymbol{H}^{3}=\mathfrak{I}$, as the restriction of Proposition 1.1. By Lemma 1.2, $\varphi_{4}$ is well-defined and a homomorphism. We shall show that $\varphi_{4}$ is onto. Let $\alpha \in\left(F_{4}\right)^{\sigma, \gamma}$. Since $\left(F_{4}\right)^{\sigma, \gamma} \subset\left(F_{4}\right)^{\gamma}$, there exist $p \in S p(1)$ and $A \in S p(3)$ such that $\alpha=\varphi(p, A)$ (Proposition 1.1). From $\sigma \alpha \sigma=\alpha$, we have $\varphi\left(p, I_{1} A I_{1}\right)=\varphi(p, A)$ (Lemma 1.2). Hence,

$$
\left\{\begin{array} { l } 
{ p = p } \\
{ I _ { 1 } A I _ { 1 } = A }
\end{array} \text { or } \left\{\begin{array}{l}
p=-p \\
I_{1} A I_{1}=-A
\end{array} .\right.\right.
$$


The latter case is impossible because $p=0$ is false. In the former case, from \begin{tabular}{c}
$I_{1} A I_{1}=A$, we have $A=\left(\begin{array}{c|cc}q & 0 & 0 \\
\hline 0 & \\
0 & B\end{array}\right), q \in S p(1), B \in S p(2)$. Hence, $\alpha=$ \\
\hline $\left.\begin{array}{lll}q & 0 & 0 \\
\hline 0\end{array}\right)$
\end{tabular} $\varphi\left(q,\left(\begin{array}{c|cc}q & 0 & 0 \\ \hline 0 & B \\ 0 & B\end{array}\right)\right)=\varphi_{4}(p, q, B)$, that is, $\varphi_{4}$ is onto. And $\operatorname{Ker} \varphi_{4}=\{(1,1, E)$, $(-1,-1,-E)\}=Z_{2}$. Thus, we have the required isomorphism $(S p(1) \times S p(1) \times$ $S p(2)) / Z_{2} \cong\left(F_{4}\right)^{\sigma, \gamma}$.

\section{Group $E_{6}$}

We use the same notation as in [1], [2] or [5] (however, some will be rewritten). For example, the complex exceptional Jordan algebra $\mathfrak{J}^{C}=\left\{X \in M\left(3, \mathfrak{C}^{C}\right) \mid\right.$ $\left.X^{*}=X\right\}$, the Freudenthal multiplication $X \times Y$ and the Hermitian inner product $\langle X, Y\rangle$, the group $E_{6}=\left\{\alpha \in \operatorname{Iso}_{C}\left(\mathfrak{J}^{C}\right) \mid \alpha X \times \alpha Y=\tau \alpha \tau(X \times Y),\langle\alpha X, \alpha Y\rangle=\right.$ $\langle X, Y\rangle\}$, and the natural inclusion $F_{4} \subset E_{6}$.

Proposition 2.1. $\left(E_{6}\right)^{\gamma} \cong(S p(1) \times S U(6)) / Z_{2}, Z_{2}=\{(1, E),(-1,-E)\}$.

Proof. The isomorphism is induced by the homomorphism $\varphi: S p(1) \times$ $S U(6) \rightarrow\left(E_{6}\right)^{\gamma}, \varphi(p, A)(M+a)=k_{J}^{-1}\left(A k_{J}(M)^{t} A\right)+p a k^{-1}\left(A^{*}\right), M+\boldsymbol{a} \in \mathfrak{I}(3, \boldsymbol{H})^{C}$ $\oplus\left(\boldsymbol{H}^{3}\right)^{C}=\mathfrak{J}^{C}$. (In detail, see [3], [5].)

Lemma 2.2. $\quad \varphi: S p(1) \times S U(6) \rightarrow\left(E_{6}\right)^{\gamma}$ of Proposition 2.1 satisfies $\sigma \varphi(p, A) \sigma=$ $\varphi\left(p, I_{2} A I_{2}\right)$, where $I_{2}=\operatorname{diag}(-1,-1,1,1,1,1)$.

Proof. From $\sigma=\varphi\left(-1, I_{2}\right)$, we have the required one.

Now, we shall determine the group structure of $\left(E_{6}\right)^{\sigma, \gamma}=\left(\left(E_{6}\right)^{\gamma}\right)^{\sigma}=\left(\left(E_{6}\right)^{\sigma}\right)^{\gamma}=$ $\left(E_{6}\right)^{\sigma} \cap\left(E_{6}\right)^{\gamma}$.

THEOREM 2.3. $\left(E_{6}\right)^{\sigma, \gamma} \cong(S p(1) \times S(U(2) \times U(4))) / Z_{2}, Z_{2}=\{(1, E)$, $(-1,-E)\}$.

Proof. We define a map $\varphi_{6}: S p(1) \times S(U(2) \times U(4)) \rightarrow\left(E_{6}\right)^{\sigma, \gamma}$ by

$$
\varphi_{6}(p, A)(M+a)=k_{J}^{-1}\left(A k_{J}(M)^{t} A\right)+p a k^{-1}\left(A^{*}\right),
$$

$\boldsymbol{M}+\boldsymbol{a} \in \mathfrak{J}(3, \boldsymbol{H})^{C} \oplus\left(\boldsymbol{H}^{3}\right)^{C}=\mathfrak{J}^{C}$, as the restriction of $\varphi$ of Proposition 2.1. By Lemma 2.2, $\varphi_{6}$ is well-defined and a homomorphism. We shall show that $\varphi_{6}$ is 
onto. Let $\alpha \in\left(E_{6}\right)^{\sigma, \gamma}$. Since $\left(E_{6}\right)^{\sigma, \gamma} \subset\left(E_{6}\right)^{\gamma}$, there exist $p \in S p(1)$ and $A \in S U(6)$ such that $\alpha=\varphi(p, A)$ (Proposition 2.1). From $\sigma \alpha \sigma=\alpha$, we have $\varphi\left(p, I_{2} A I_{2}\right)=$ $\varphi(p, A)$ (Lemma 2.2). Hence,

$$
\left\{\begin{array} { l } 
{ p = p } \\
{ I _ { 2 } A I _ { 2 } = A }
\end{array} \text { or } \left\{\begin{array}{l}
p=-p \\
I_{2} A I_{2}=-A
\end{array} .\right.\right.
$$

The latter case is impossible because $p=0$ is false. In the former case, we have $A \in S(U(2) \times U(4))$. Therefore, $\varphi_{6}$ is onto. $\operatorname{Ker} \varphi_{6}=\{(1, E),(-1,-E)\}=Z_{2}$. Thus, we have the required isomorphism $(S p(1) \times S(U(2) \times U(4))) / Z_{2} \cong\left(E_{6}\right)^{\sigma, \gamma}$.

\section{Group $E_{7}$}

We use the same notation as in [1], [4] or [5] (however, some will be rewritten). For example, the Freudenthal $C$-vector space $\mathfrak{P}^{C}=\mathfrak{J}^{C} \oplus \mathfrak{I}^{C} \oplus C \oplus C$, the Hermitian inner product $\langle P, Q\rangle$, the $C$-linear map $P \times Q: \mathfrak{P}^{C} \rightarrow \mathfrak{P}^{C}\left(P, Q \in \mathfrak{P}^{C}\right)$, the group $E_{7}=\left\{\alpha \in \operatorname{Iso}_{C}\left(\mathfrak{P}^{C}\right) \mid \alpha(X \times Y) \alpha^{-1}=\alpha P \times \alpha Q,\langle\alpha P, \alpha Q\rangle=\langle P, Q\rangle\right\}$, the natural inclusion $E_{6} \subset E_{7}$ and elements $\sigma, \sigma^{\prime} \in F_{4} \subset E_{6} \subset E_{7}, \lambda \in E_{7}$.

We shall consider the following subgroup of $F_{4}$.

$$
\left(\left(F_{4}\right)^{\sigma, \gamma}\right)_{F_{1}(h)}=\left\{\alpha \in\left(F_{4}\right)^{\sigma, \gamma} \mid \alpha F_{1}(h)=F_{1}(h) \text { for all } h \in \boldsymbol{H}\right\} .
$$

Proposition 3.1. $\left(\left(F_{4}\right)^{\sigma, \gamma}\right)_{F_{1}(h)} \cong S p(1) \times S p(1)(=\operatorname{Spin}(4))$.

Proof. We define a map $\varphi: S p(1) \times S p(1) \rightarrow\left(\left(F_{4}\right)^{\sigma, \gamma}\right)_{F_{1}(h)}$ by

$$
\varphi(p, q)(M+\boldsymbol{a})=\left(\begin{array}{c|cc}
q & 0 & 0 \\
\hline 0 & E \\
0 & E
\end{array}\right) M\left(\begin{array}{c|cc}
q & 0 & 0 \\
\hline 0 & E \\
0 & E
\end{array}\right)^{*}+p a\left(\begin{array}{c|cc}
q & 0 & 0 \\
\hline 0 & & E \\
0 & E
\end{array}\right)^{*}
$$

as the restriction of $\varphi_{4}$ of Theorem 1.3. By $F_{1}(h)=\left(\begin{array}{lll}0 & 0 & 0 \\ 0 & 0 & h \\ 0 & \bar{h} & 0\end{array}\right)+\boldsymbol{O}, \varphi$ is welldefined and homomorphism. We shall show that $\varphi$ is onto. Let $\alpha \in\left(\left(F_{4}\right)^{\sigma, \gamma}\right)_{F_{1}(h)}$. Since $\left(\left(F_{4}\right)^{\sigma, \gamma}\right)_{F_{1}(h)} \subset\left(F_{4}\right)^{\sigma, \gamma}$, there exist $p, q \in S p(1)$ and $B \in S p(2)$ such that $\alpha=$ $\varphi_{4}(p, q, B)$ (Theorem 1.3). From $\alpha F_{1}(h)=F_{1}(h)$, we have $B\left(\begin{array}{ll}0 & h \\ \bar{h} & 0\end{array}\right) B^{*}=\left(\begin{array}{ll}0 & h \\ \bar{h} & 0\end{array}\right)$,
so that

$$
\alpha=\varphi_{4}(p, q, E) \quad \text { or } \quad \alpha=\varphi_{4}(p, q,-E) \text {. }
$$

In the former case, we have $\alpha=\varphi_{4}(p, q, E)=\varphi(p, q)$. In the latter case, we have 


$$
\begin{aligned}
\alpha & =\varphi_{4}(p, q,-E)=\varphi_{4}(-p,-q, E) \varphi_{4}(-1,-1,-E) \\
& =\varphi_{4}(-p,-q, E) 1=\varphi(-p,-q)
\end{aligned}
$$

Hence, $\varphi$ is onto. $\operatorname{Ker} \varphi=\{(1,1)\}$. Thus, we have the required isomorphism $S p(1) \times S p(1) \cong\left(\left(F_{4}\right)^{\sigma, \gamma}\right)_{F_{1}(h)}$.

Hereafter, in $\mathfrak{P}^{C}$, we use the following notations.

$$
\begin{gathered}
\left(F_{1}(h), 0,0,0\right)=\dot{F}_{1}(h), \quad\left(0, E_{1}, 0,1\right)=\tilde{E}_{1}, \\
\left(0, E_{1}, 0,-1\right)=\tilde{E}_{-1}, \quad\left(E_{2}+E_{3}, 0,0,0\right)=\dot{E}_{23} .
\end{gathered}
$$

We shall consider a subgroup $\left(\left(\left(E_{7}\right)^{\kappa, \mu}\right)^{\gamma}\right)_{\dot{F}_{1}(h), \tilde{E}_{1}, \tilde{E}_{-1}, \dot{E}_{23}}$ of $E_{7}$.

LEMMA 3.2. The Lie algebra $\left(\left(\left(\mathfrak{e}_{7}\right)^{\kappa, \mu}\right)^{\gamma}\right)_{\dot{F}_{1}(h), \tilde{E}_{1}, \tilde{E}_{-1}, \dot{E}_{23}}$ of the group $\left(\left(\left(E_{7}\right)^{\kappa, \mu}\right)^{\gamma}\right)_{\dot{F}_{1}(h), \tilde{E}_{1}, \tilde{E}_{-1}, \dot{E}_{23}}$ is given by

$$
\begin{aligned}
& \left(\left(\left(\mathfrak{e}_{7}\right)^{\kappa, \mu}\right)^{\gamma}\right)_{\dot{F}_{1}(h), \tilde{E}_{1}, \tilde{E}_{-1}, \dot{E}_{23}} \\
& \quad=\left\{\Phi\left(\left(\begin{array}{c|c}
0 & 0 \\
\hline 0 & D_{4}^{\prime}
\end{array}\right), 0,0,0\right) \mid\left(\begin{array}{c|c}
0 & 0 \\
\hline 0 & D_{4}^{\prime}
\end{array}\right) \in \mathfrak{s o}(8), D_{4}^{\prime} \in \mathfrak{s o}(4)\right\} .
\end{aligned}
$$

In particular, we have

$$
\operatorname{dim}\left(\left(\left(\left(\mathfrak{e}_{7}\right)^{\kappa, \mu}\right)^{\gamma}\right)_{\dot{F}_{1}(h), \tilde{E}_{1}, \tilde{E}_{-1}, \dot{E}_{23}}\right)=6
$$

Hereafter, $\left(\begin{array}{c|c}0 & 0 \\ \hline 0 & D_{4}^{\prime}\end{array}\right)$ will be denoted by $D_{4}^{\prime}$, and also $\Phi\left(D_{4}^{\prime}, 0,0,0\right)$ will be denoted by $\Phi_{4}$.

Proposition 3.3. $\quad\left(\left(\left(E_{7}\right)^{\kappa, \mu}\right)^{\gamma}\right)_{\dot{F}_{1}(h), \tilde{E}_{1}, \tilde{E}_{-1}, \dot{E}_{23}}=\left(\left(F_{4}\right)^{\sigma, \gamma}\right)_{F_{1}(h)}$.

Proof. Let $\alpha \in\left(\left(F_{4}\right)^{\sigma, \gamma}\right)_{F_{1}(h)}$. Since $\left(\left(F_{4}\right)^{\sigma, \gamma}\right)_{F_{1}(h)} \subset\left(F_{4}\right)^{\sigma}=\left(F_{4}\right)_{E_{1}}$ (as for $\left(F_{4}\right)^{\sigma}$ $=\left(F_{4}\right)_{E_{1}}$, see [3], [5]), we see $\alpha E_{1}=E_{1}$. As a result, because $\kappa$ and $\mu$ are defined using by $E_{1}$ (see [1], [4] or [5]), we see that $\kappa \alpha=\alpha \kappa$ and $\mu \alpha=\alpha \mu$. From $\alpha E=E$ (see [3], [5]), we have $\alpha\left(E_{2}+E_{3}\right)=E_{2}+E_{3}$. Hence, $\alpha \dot{E}_{23}=\dot{E}_{23}$. Moreover, from $\alpha(0,0,0,1)=(0,0,0,1)$ (see [4], [5]), we have $\alpha \tilde{E}_{1}=\tilde{E}_{1}$ and $\alpha \tilde{E}_{-1}=$ $\tilde{E}_{-1}$. Obviously $\alpha \dot{F}_{1}(h)=\dot{F}_{1}(h)$. Thus, $\alpha \in\left(\left(\left(E_{7}\right)^{\kappa, \mu}\right)^{\gamma}\right)_{\dot{F}_{1}(h), \tilde{E}_{1}, \tilde{E}_{-1}, \dot{E}_{23}}$. Conversely,

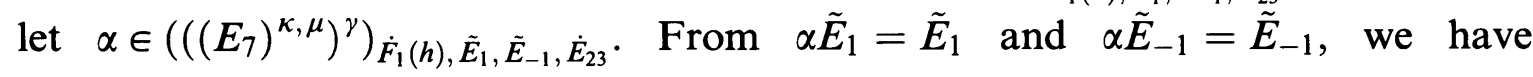


Fixed points subgroups by two involutive automorphisms

$\alpha\left(0, E_{1}, 0,0\right)=\left(0, E_{1}, 0,0\right)$ and $\alpha(0,0,0,1)=(0,0,0,1)$. Hence, $\alpha \in\left(\left(E_{6}\right)^{\gamma}\right)_{F_{1}(h), E_{1}, E_{2}+E_{3}}$ (see [4], [5]). Thus, $\left(\left(\left(F_{4}\right)_{E_{1}}\right)^{\gamma}\right)_{F_{1}(h)}=\left(\left(F_{4}\right)^{\sigma, \gamma}\right)_{F_{1}(h)}$. Therefore, the proof of this proposition is completed.

Next, we shall consider the following subgroup of $F_{4}$.

$$
\left(\left(F_{4}\right)^{\sigma, \gamma}\right)_{F_{1}\left(h e_{4}\right)}=\left\{\alpha \in\left(F_{4}\right)^{\sigma, \gamma} \mid \alpha F_{1}\left(h e_{4}\right)=F_{1}\left(h e_{4}\right) \text { for all } h \in \boldsymbol{H}\right\} .
$$

PROPOSITION 3.4. $\left(\left(F_{4}\right)^{\sigma, \gamma}\right)_{F_{1}\left(h e_{4}\right)} \cong S p(2)(=\operatorname{Spin}(5))$.

Proof. We define a map $\varphi: S p(2) \rightarrow\left(\left(F_{4}\right)^{\sigma, \gamma}\right)_{F_{1}\left(h e_{4}\right)}$ by

$$
\varphi(B)(M+a)=\left(\begin{array}{c|cc}
1 & 0 & 0 \\
\hline 0 & B \\
0 & B
\end{array}\right) M\left(\begin{array}{c|cc}
1 & 0 & 0 \\
\hline 0 & B \\
0 & B
\end{array}\right)^{*}+a\left(\begin{array}{l|ll}
1 & 0 & 0 \\
\hline 0 & B \\
0 & B
\end{array}\right)^{*},
$$

as the restriction of $\varphi_{4}$ of Theorem 1.3. Obviously $\varphi$ is well-defined and homomorphism. We shall show that $\varphi$ is onto. Let $\alpha \in\left(\left(F_{4}\right)^{\sigma, \gamma}\right)_{F_{1}\left(h_{4}\right)}$. Since $\left(\left(F_{4}\right)^{\sigma, \gamma}\right)_{F_{1}\left(h e_{4}\right)} \subset\left(F_{4}\right)^{\sigma, \gamma}$, there exist $p, q \in S p(1)$ and $B \in S p(2)$ such that $\alpha=$ $\varphi_{4}(p, q, B)$ (Theorem 1.3). From $\alpha F_{1}\left(h e_{4}\right)=F_{1}\left(h e_{4}\right)(=O+(h, 0,0))$, we have $p h \bar{q}=h(h \in \boldsymbol{H})$, so that

$$
\alpha=\varphi_{4}(1,1, B) \quad \text { or } \quad \alpha=\varphi_{4}(-1,-1, B) .
$$

In the former case, we have $\alpha=\varphi_{4}(1,1, B)=\varphi(B)$. In the latter case, we have

$$
\begin{aligned}
\alpha & =\varphi_{4}(-1,-1, B)=\varphi_{4}(1,1,-B) \varphi_{4}(-1,-1,-E) \\
& =\varphi_{4}(1,1,-B) 1=\varphi(-B) .
\end{aligned}
$$

Hence, $\varphi$ is onto. $\operatorname{Ker} \varphi=\{E\}$. Thus, we have the required isomorphism $\operatorname{Sp}(2) \cong$ $\left(\left(F_{4}\right)^{\sigma, \gamma}\right)_{F_{1}\left(h e_{4}\right)}$.

Then, we have the following proposition.

Proposition 3.5. $\quad\left(\left(\left(E_{7}\right)^{\kappa, \mu}\right)^{\gamma}\right)_{\dot{F}_{1}\left(h e_{4}\right), \tilde{E}_{1}, \tilde{E}_{-1}, \dot{E}_{23}}=\left(\left(F_{4}\right)^{\sigma, \gamma}\right)_{F_{1}\left(h e_{4}\right)}$.

Proof. This proof is in the way similar to Proposition 3.3.

We shall consider the subgroup $\left(\left(\left(E_{7}\right)^{\kappa, \mu}\right)^{\gamma}\right)_{\dot{F}_{1}\left(h e_{4}\right), \tilde{E}_{1}, \tilde{E}_{-1}}$ of $E_{7}$.

LEMMA 3.6. The Lie algebra $\left(\left(\left(\mathrm{e}_{7}\right)^{\kappa, \mu}\right)^{\gamma}\right)_{\dot{F}_{1}\left(e_{4}\right), \tilde{E}_{1}, \tilde{E}_{-1}}$ of the group $\left(\left(\left(E_{7}\right)^{\kappa, \mu}\right)^{\gamma}\right)_{\dot{F}_{1}\left(h e_{4}\right), \tilde{E}_{1}, \tilde{E}_{-1}}$ is given by 


$$
\begin{aligned}
\left(\left(\left(\mathfrak{e}_{7}\right)^{\kappa, \mu}\right)^{\gamma}\right)_{\dot{F}_{1}\left(h e_{4}\right), \tilde{E}_{1}, \tilde{E}_{-1}} & \\
= & \left\{\Phi\left(\left(\begin{array}{c|c}
D_{4} & 0 \\
\hline 0 & 0
\end{array}\right)+\tilde{A}_{1}(p)+i\left(\begin{array}{ccc}
0 & 0 & 0 \\
0 & \varepsilon & q \\
0 & \bar{q} & -\varepsilon
\end{array}\right)^{\sim}, 0,0,0\right) \mid\left(\begin{array}{c|c}
D_{4} & 0 \\
\hline 0 & 0
\end{array}\right) \in \mathfrak{s o}(8),\right. \\
& \left.D_{4} \in \mathfrak{s o}(4), \varepsilon \in \boldsymbol{R}, p, q \in \boldsymbol{H}\right\} .
\end{aligned}
$$

In particular, we have

$$
\operatorname{dim}\left(\left(\left(\left(\mathfrak{e}_{7}\right)^{\kappa, \mu}\right)^{\gamma}\right)_{\dot{F}_{1}\left(h e_{4}\right), \tilde{E}_{1}, \tilde{E}_{-1}}\right)=15
$$

Hereafter, $\left(\begin{array}{c|c}D_{4} & 0 \\ \hline 0 & 0\end{array}\right)$ will be denoted by $D_{4}$.

Lemma 3.7. (1) For $a \in \boldsymbol{H}$, we define a map $\tilde{\alpha}_{1}(a)$ of $\mathfrak{J}^{C}$ by

$$
\begin{aligned}
& \left\{\begin{array}{l}
\xi_{1}^{\prime}=\xi_{1} \\
\xi_{2}^{\prime}=\frac{\xi_{2}-\xi_{3}}{2}+\frac{\xi_{2}+\xi_{3}}{2} \cos |a|+i \frac{\left(a, x_{1}\right)}{|a|} \sin |a| \\
\xi_{3}^{\prime}=-\frac{\xi_{2}-\xi_{3}}{2}+\frac{\xi_{2}+\xi_{3}}{2} \cos |a|+i \frac{\left(a, x_{1}\right)}{|a|} \sin |a|
\end{array}\right. \\
& \left\{\begin{array}{l}
x_{1}^{\prime}=x_{1}+i \frac{\left(\xi_{2}+\xi_{3}\right) a}{|a|} \sin |a|-\frac{2\left(a, x_{1}\right) a}{|a|^{2}}\left(\sin \frac{|a|}{2}\right)^{2} \\
x_{2}^{\prime}=x_{2} \cos \frac{|a|}{2}+i \frac{\overline{x_{3} a}}{|a|} \sin \frac{|a|}{2} \\
x_{3}^{\prime}=x_{3} \cos \frac{|a|}{2}+i \frac{\overline{a x_{2}}}{|a|} \sin \frac{|a|}{2}
\end{array}\right.
\end{aligned}
$$

Then, $\tilde{\alpha}_{1}(a) \in\left(\left(\left(E_{7}\right)^{\kappa, \mu}\right)^{\gamma}\right)_{\dot{F}_{1}\left(h e_{4}\right), \tilde{E}_{1}, \tilde{E}_{-1}}$.

(2) For $t \in \boldsymbol{R}$, we define a map $\tilde{\alpha}_{23}(t)$ of $\mathfrak{J}^{C}$ by

$$
\tilde{\alpha}_{23}(t)\left(\begin{array}{ccc}
\xi_{1} & x_{3} & \bar{x}_{2} \\
\bar{x}_{3} & \xi_{2} & x_{1} \\
x_{2} & \bar{x}_{1} & \xi_{3}
\end{array}\right)=\left(\begin{array}{ccc}
\xi_{1} & e^{i t / 2} x_{3} & e^{-i t / 2} \bar{x}_{2} \\
e^{i t / 2} \bar{x}_{3} & e^{i t} \xi_{2} & x_{1} \\
e^{-i t / 2} x_{2} & \bar{x}_{1} & e^{-i t \xi_{3}}
\end{array}\right) .
$$

Then, $\tilde{\alpha}_{23}(t) \in\left(\left(\left(E_{7}\right)^{\kappa, \mu}\right)^{\gamma}\right)_{\dot{F}_{1}\left(h e_{4}\right), \tilde{E}_{1}, \tilde{E}_{-1}}$.

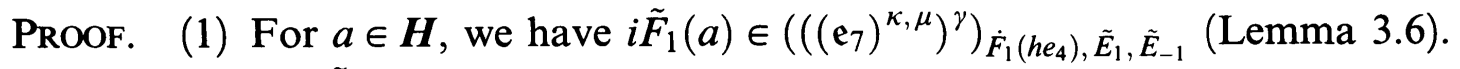
Hence, $\tilde{\alpha}_{1}(a)=\exp i \tilde{F}_{1}(a) \in\left(\left(\left(E_{7}\right)^{\kappa, \mu}\right)^{\gamma}\right)_{\dot{F}_{1}\left(h e_{4}\right), \tilde{E}_{1}, \tilde{E}_{-1}}$. 


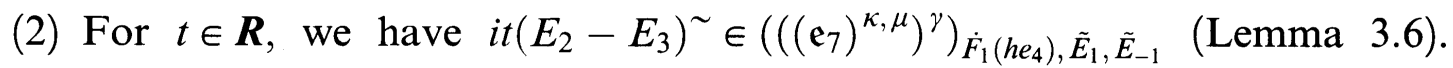
Hence, $\tilde{\alpha}_{23}(t)=\exp i t\left(E_{2}-E_{3}\right)^{\sim} \in\left(\left(\left(E_{7}\right)^{\kappa, \mu}\right)^{\gamma}\right)_{\dot{F}_{1}\left(h e_{4}\right), \tilde{E}_{1}, \tilde{E}_{-1}}$.

We define a 6 dimensional $\boldsymbol{R}$-vector space $V^{6}$ by

$$
\begin{aligned}
V^{6} & =\left\{P \in \mathfrak{P}^{C} \mid \kappa P=P, \mu \tau \lambda P=P, \gamma P=P,\left\langle P, \tilde{E}_{1}\right\rangle=0,\left\langle P, \tilde{E}_{-1}\right\rangle=0\right\} \\
& =\left\{P=\left(\left(\begin{array}{ccc}
0 & 0 & 0 \\
0 & \xi & h \\
0 & \bar{h} & -\tau \xi
\end{array}\right), 0,0,0\right) \mid \xi \in C, h \in \boldsymbol{H}\right\}
\end{aligned}
$$

with the norm (see [5] for the definition of $\{$,$\} 's)$

$$
(P, P)_{\mu}=\frac{1}{2}\{\mu P, P\}=\frac{1}{2}(\mu P, \lambda P)=(\tau \xi) \xi+\bar{h} h .
$$

Then, $S^{5}=\left\{P \in V^{6} \mid(P, P)_{\mu}=1\right\}$ is a 5 dimensional sphere.

LEMMA 3.8. $\quad\left(\left(\left(E_{7}\right)^{\kappa, \mu}\right)^{\gamma}\right)_{\dot{F}_{1}\left(h e_{4}\right), \tilde{E}_{1}, \tilde{E}_{-1}} / \operatorname{Spin}(5) \simeq S^{5}$. In particular, $\left(\left(\left(E_{7}\right)^{\kappa, \mu}\right)^{\gamma}\right)_{\dot{F}_{1}\left(h e_{4}\right), \tilde{E}_{1}, \tilde{E}_{-1}}$ is connected.

Proof. Since $E_{7}$ is commutative with $\tau \lambda$, the group $\left(\left(\left(E_{7}\right)^{\kappa, \mu}\right)^{\gamma}\right)_{\dot{F}_{1}\left(h e_{4}\right), \tilde{E}_{1}, \tilde{E}_{-1}}$ acts on $S^{5}$. We shall show that this action is transitive. To show this, it is sufficient to show that any element $P \in S^{5}$ can be transformed to $\left(i\left(E_{2}+E_{3}\right), 0,0,0\right) \in S^{5}$ under the action of $\left(\left(\left(E_{7}\right)^{\kappa, \mu}\right)^{\gamma}\right)_{\dot{F}_{1}\left(h e_{4}\right), \tilde{E}_{1}, \tilde{E}_{-1}}$. Now, for a given

$$
P=\left(\left(\begin{array}{ccc}
0 & 0 & 0 \\
0 & \xi & h \\
0 & \bar{h} & -\tau \xi
\end{array}\right), 0,0,0\right) \in S^{5},
$$

choose $t \in \boldsymbol{R}$ such that $e^{i t} \xi \in \boldsymbol{R}$. For this $t \in \boldsymbol{R}$, operate $\tilde{\alpha}_{23}(t)($ Lemma $3.7(2)) \in$ $\left(\left(\left(E_{7}\right)^{\kappa, \mu}\right)^{\gamma}\right)_{\dot{F}_{1}\left(h e_{4}\right), \tilde{E}_{1}, \tilde{E}_{-1}}$ on $P$. Then, we have

$$
\tilde{\alpha}_{23}(t) P=\left(\left(\begin{array}{ccc}
0 & 0 & 0 \\
0 & r & h \\
0 & \bar{h} & -r
\end{array}\right), 0,0,0\right)=P_{1}, \quad r \in \boldsymbol{R} .
$$

In the case of $h \neq 0$, operate $\tilde{\alpha}_{1}(\pi h / 2|h|)($ Lemma 3.7(1)) $\epsilon$ $\left(\left(\left(E_{7}\right)^{\kappa, \mu}\right)^{\gamma}\right)_{\dot{F}_{1}\left(h e_{4}\right), \tilde{E}_{1}, \tilde{E}_{-1}}$ on $P_{1}$. Then, we have

$$
\tilde{\alpha}_{1}\left(\frac{\pi h}{2|h|}\right) P_{1}=\left(\left(\begin{array}{ccc}
0 & 0 & 0 \\
0 & \xi^{\prime} & 0 \\
0 & 0 & -\tau \xi^{\prime}
\end{array}\right), 0,0,0\right)=P_{2} \in S^{5}, \quad \xi^{\prime} \in C .
$$


Here, from $\left(\tau \xi^{\prime}\right) \xi^{\prime}=1, \xi^{\prime} \in C$, we can put $\xi^{\prime}=e^{i \theta}, 0 \leq \theta<2 \pi$. Operate $\tilde{\alpha}_{23}(-\theta)$ on $P_{2}$. Then,

$$
\tilde{\alpha}_{23}(-\theta) P_{2}=\left(E_{2}-E_{3}, 0,0,0\right)=P_{3} .
$$

Moreover, operate $\tilde{\alpha}_{23}(\pi / 2)$ on $P_{3}$,

$$
\tilde{\alpha}_{23}\left(\frac{\pi}{2}\right) P_{3}=\left(i\left(E_{2}+E_{3}\right), 0,0,0\right)=i \dot{E}_{23}
$$

This shows the transitivity. The isotropy subgroup $\left(\left(\left(E_{7}\right)^{\kappa, \mu}\right)^{\gamma}\right)_{\dot{F}_{1}\left(h e_{4}\right), \tilde{E}_{1}, \tilde{E}_{-1}}$ at $\dot{E}_{23}$ is $\left(\left(\left(E_{7}\right)^{\kappa, \mu}\right)^{\gamma}\right)_{\dot{F}_{1}\left(h e_{4}\right), \tilde{E}_{1}, \tilde{E}_{-1}, \dot{E}_{23}}=\operatorname{Sp}(2)$ (Propositions 3.4, 3.5) $=\operatorname{Spin}(5)$. Therefore, we have the homeomorphism $\left(\left(\left(E_{7}\right)^{\kappa, \mu}\right)^{\gamma}\right)_{\dot{F}_{1}\left(h e_{4}\right), \tilde{E}_{1}, \tilde{E}_{-1}} / \operatorname{Spin}(5) \simeq S^{5}$.

PROPOSITION 3.9. $\quad\left(\left(\left(E_{7}\right)^{\kappa, \mu}\right)^{\gamma}\right)_{\dot{F}_{1}\left(h e_{4}\right), \tilde{E}_{1}, \tilde{E}_{-1}} \cong \operatorname{Spin}(6)$.

ProOF. Since $\left(\left(\left(E_{7}\right)^{\kappa, \mu}\right)^{\gamma}\right)_{\dot{F}_{1}\left(h e_{4}\right), \tilde{E}_{1}, \tilde{E}_{-1}}$ is connected (Lemma 3.8), we can define a homormorphism $\pi:\left(\left(\left(E_{7}\right)^{\kappa, \mu}\right)^{\gamma}\right)_{\dot{F}_{1}\left(h e_{4}\right), \tilde{E}_{1}, \tilde{E}_{-1}} \rightarrow S O(6)=S O\left(V^{6}\right)$ by

$$
\pi(\alpha)=\alpha \mid V^{6} \text {. }
$$

It is not difficult to see that $\operatorname{Ker} \varphi=\{1, \sigma\}=Z_{2}$. Since $\operatorname{dim}\left(\left(\left(\left(E_{7}\right)^{\kappa, \mu}\right)^{\gamma}\right)_{\dot{F}_{1}\left(h_{4}\right), \tilde{E}_{1}, \tilde{E}_{-1}}\right)=15$ (Lemma 3.6) $=\operatorname{dim}(\mathfrak{s o}(6)), \pi$ is onto. Hence, $\left(\left(\left(E_{7}\right)^{\kappa, \mu}\right)^{\gamma}\right)_{\dot{F}_{1}\left(h e_{4}\right), \tilde{E}_{1}, \tilde{E}_{-1}} \boldsymbol{Z}_{2} \cong S O(6)$. Therefore, $\left(\left(\left(E_{7}\right)^{\kappa, \mu}\right)^{\gamma}\right)_{\dot{F}_{1}\left(h e_{4}\right), \tilde{E}_{1}, \tilde{E}_{-1}}$ is isomorphism to $\operatorname{Spin}(6)$ as a double covering group of $S O(6)$.

We shall consider a subgroup $\left(\left(\left(E_{7}\right)^{\kappa, \mu}\right)^{\gamma}\right)_{\dot{F}_{1}\left(h e_{4}\right), \tilde{E}_{1}}$ of $E_{7}$.

LEMMA 3.10. The Lie algebra $\left(\left(\left(\mathfrak{e}_{7}\right)^{\kappa, \mu}\right)^{\gamma}\right)_{\dot{F}_{1}\left(h e_{4}\right), \tilde{E}_{1}}$ of the group $\left(\left(\left(E_{7}\right)^{\kappa, \mu}\right)^{\gamma}\right)_{\dot{F}_{1}\left(h e_{4}\right), \tilde{E}_{1}}$ is given by

$$
\begin{aligned}
\left(\left(\left(\mathrm{e}_{7}\right)^{\kappa, \mu}\right)^{\gamma}\right)_{\dot{F}_{1}\left(h e_{4}\right), \tilde{E}_{1}} & \left\{\Phi\left(D_{4}+\tilde{A}_{1}(p)+i\left(\begin{array}{ccc}
0 & 0 & 0 \\
0 & \varepsilon & q \\
0 & \bar{q} & -\varepsilon
\end{array}\right),\left(\begin{array}{ccc}
0 & 0 & 0 \\
0 & \alpha & i x \\
0 & \overline{i x} & \tau \alpha
\end{array}\right),-\tau\left(\begin{array}{ccc}
0 & 0 & 0 \\
0 & \alpha & i x \\
0 & \overline{i x} & \tau \alpha
\end{array}\right), 0\right)\right. \\
& \left.\mid D_{4} \in \mathfrak{s o}(4) \subset \mathfrak{s o}(8), \varepsilon \in \boldsymbol{R}, \alpha \in C, p, q, x \in \boldsymbol{H}\right\} .
\end{aligned}
$$

In particular, we have

$$
\operatorname{dim}\left(\left(\left(\left(\mathrm{e}_{7}\right)^{\kappa, \mu}\right)^{\gamma}\right)_{\dot{F}_{1}\left(h e_{4}\right), \tilde{E}_{1}}\right)=21
$$

Lemma 3.11. For $a \in \boldsymbol{R}$, we define maps $\alpha_{k}(a), k=2,3$ of $\mathfrak{P}^{C}$ by 


$$
\alpha_{k}(a)\left(\begin{array}{c}
X \\
Y \\
\xi \\
\eta
\end{array}\right)=\left(\begin{array}{c}
\left(1+(\cos a-1) p_{k}\right) X-2(\sin a) E_{k} \times Y+\eta(\sin a) E_{k} \\
2(\sin a) E_{k} \times X+\left(1+(\cos a-1) p_{k}\right) Y-\xi(\sin a) E_{k} \\
\left((\sin a) E_{k}, Y\right)+(\cos a) \xi \\
\left(-(\sin a) E_{k}, X\right)+(\cos a) \eta
\end{array}\right),
$$

where $p_{k}: \mathfrak{J}^{C} \rightarrow \mathfrak{J}^{C}$ is defined by

$$
p_{k}(X)=\left(X, E_{k}\right) E_{k}+4 E_{k} \times\left(E_{k} \times X\right), \quad X \in \mathfrak{J}^{C} .
$$

Then, $\alpha_{k} \in E_{7}$ and $\alpha_{2}(a), \alpha_{3}(b)(a, b \in \boldsymbol{R})$ commute with each other.

ProOF. For $\Phi_{k}(a)=\Phi\left(0, a E_{k},-a E_{k}, 0\right) \in \mathfrak{e}_{7}$, we have $\alpha_{k}(a)=\exp \Phi_{k}(a) \in E_{7}$. Since $\left[\Phi_{2}(a), \Phi_{3}(b)\right]=0, \alpha_{2}(a)$ and $\alpha_{3}(b)$ are commutative.

We define a 7 dimensional $\boldsymbol{R}$-vector space $V^{7}$ by

$$
\begin{aligned}
V^{7} & =\left\{P \in \mathfrak{P}^{C} \mid \kappa P=P, \mu \tau \lambda P=P, \gamma P=P,\left\langle P, \tilde{E}_{1}\right\rangle=0\right\} \\
& =\left\{P=\left(\left(\begin{array}{ccc}
0 & 0 & 0 \\
0 & \xi & h \\
0 & \bar{h} & -\tau \xi
\end{array}\right),\left(\begin{array}{ccc}
\text { in } & 0 & 0 \\
0 & 0 & 0 \\
0 & 0 & 0
\end{array}\right), 0,-i \eta\right) \mid \xi \in C, h \in \boldsymbol{H}, \eta \in \boldsymbol{R}\right\}
\end{aligned}
$$

with the norm

$$
(P, P)_{\mu}=\frac{1}{2}(\mu P, \lambda P)=(\tau \xi) \xi+\bar{h} h+\eta^{2} .
$$

Then, $S^{6}=\left\{P \in V^{7} \mid(P, P)_{\mu}=1\right\}$ is a 6 dimensional sphere.

LEMMA 3.12. $\quad\left(\left(\left(E_{7}\right)^{\kappa, \mu}\right)^{\gamma}\right)_{\dot{F}_{1}\left(h e_{4}\right), \tilde{E}_{1}} / \operatorname{Spin}(6) \simeq S^{6}$. In particular, $\left(\left(\left(E_{7}\right)^{\kappa, \mu}\right)^{\gamma}\right)_{\dot{F}_{1}\left(h e_{4}\right), \tilde{E}_{1}}$ is connected.

Proof. The group $\left(\left(\left(E_{7}\right)^{\kappa, \mu}\right)^{\gamma}\right)_{\dot{F}_{1}\left(h e_{4}\right), \tilde{E}_{1}}$ acts on $S^{6}$. We shall show that this action is transitive. To show this, it is sufficient to show that any element $P \in S^{6}$ can be transformed to $\left(0,-i E_{1}, 0, i\right) \in S^{6}$ under the action of $\left(\left(\left(E_{7}\right)^{\kappa, \mu}\right)^{\gamma}\right)_{\dot{F}_{1}\left(h e_{4}\right), \tilde{E}_{1}}$. Now, for a given

$$
P=\left(\left(\begin{array}{ccc}
0 & 0 & 0 \\
0 & \xi & h \\
0 & \bar{h} & -\tau \xi
\end{array}\right),\left(\begin{array}{ccc}
i \eta & 0 & 0 \\
0 & 0 & 0 \\
0 & 0 & 0
\end{array}\right), 0,-i \eta\right) \in S^{6},
$$

choose $a \in \boldsymbol{R}, 0 \leq a<\pi / 2$ such that $\tan 2 a=\frac{i 2 \eta}{\tau \xi-\xi}$ (if $\tau \xi-\xi=0$, then let 
$a=\pi / 4) . \quad$ Operate $\quad \alpha_{23}(a):=\alpha_{2}(a) \alpha_{3}(a)=\exp \left(\Phi\left(0, a\left(E_{2}+E_{3}\right),-a\left(E_{2}+E_{3}\right), 0\right)\right)$ (Lemma 3.11) $\in\left(\left(\left(E_{7}\right)^{\kappa, \mu}\right)^{\gamma}\right)_{\dot{F}_{1}\left(h_{4}\right), \tilde{E}_{1}}$ (Lemma 3.10) on $P$. Then, the $\eta$-term of $\alpha_{23}(a) P$ is $(1 / 2)(\xi-\tau \xi) \sin 2 a+i \eta \cos 2 a=0$. Hence,

$$
\alpha_{23}(a) P=\left(\left(\begin{array}{ccc}
0 & 0 & 0 \\
0 & \zeta & m \\
0 & \bar{m} & -\tau \zeta
\end{array}\right), 0,0,0\right)=P_{1} \in S^{5} \subset S^{6} .
$$

Since $\left(\left(\left(E_{7}\right)^{\kappa, \mu}\right)^{\gamma}\right)_{\dot{F}_{1}\left(h e_{4}\right), \tilde{E}_{1}, \tilde{E}_{-1}}\left(\subset\left(\left(\left(E_{7}\right)^{\kappa, \mu}\right)^{\gamma}\right)_{\dot{F}_{1}\left(h e_{4}\right), \tilde{E}_{1}}\right)$ acts transitivity on $S^{5}$ (Lemma 3.8), there exist $\beta \in\left(\left(\left(E_{7}\right)^{\kappa, \mu}\right)^{\gamma}\right)_{\dot{F}_{1}\left(h e_{4}\right), \tilde{E}_{1}, \tilde{E}_{-1}}$ such that $\beta P_{1}=\left(i\left(E_{2}+E_{3}\right), 0,0,0\right)=$ $P_{2} \in S^{5} \subset S^{6}$. Moreover, operate $\alpha_{23}(-\pi / 4)$ on $P_{2}$,

$$
\alpha_{23}\left(-\frac{\pi}{4}\right) P_{2}=\left(0,-i E_{1}, 0, i\right)=-i \tilde{E}_{-1} .
$$

This shows the transitivity. The isotropy subgroup $\left(\left(\left(E_{7}\right)^{\kappa, \mu}\right)^{\gamma}\right)_{\dot{F}_{1}\left(h e_{4}\right), \tilde{E}_{1}}$ at $\tilde{E}_{-1}$ is $\left(\left(\left(E_{7}\right)^{\kappa, \mu}\right)^{\gamma}\right)_{\dot{F}_{1}\left(h e_{4}\right), \tilde{E}_{1}, \tilde{E}_{-1}}=\operatorname{Spin}(6)$ (Proposition 3.9). Thus, we have the homeomorphism $\left(\left(\left(E_{7}\right)^{\kappa, \mu}\right)^{\gamma}\right)_{\dot{F}_{1}\left(h e_{4}\right), \tilde{E}_{1}} / \operatorname{Spin}(6) \simeq S^{6}$.

PROPOSITION 3.13. $\left(\left(\left(E_{7}\right)^{\kappa, \mu}\right)^{\gamma}\right)_{\dot{F}_{1}\left(h e_{4}\right), \tilde{E}_{1}} \cong \operatorname{Spin}(7)$.

Proof. Since $\left(\left(\left(E_{7}\right)^{\kappa, \mu}\right)^{\gamma}\right)_{\dot{F}_{1}\left(h_{4}\right), \tilde{E}_{1}}$ is connected (Lemma 3.12), we can define a homormorphism $\pi:\left(\left(\left(E_{7}\right)^{\kappa, \mu}\right)^{\gamma}\right)_{\dot{F}_{1}\left(h e_{4}\right), \tilde{E}_{1}} \rightarrow S O(7)=S O\left(V^{7}\right)$ by

$$
\pi(\alpha)=\alpha \mid V^{7}
$$

It is not difficult to see that $\operatorname{Ker} \varphi=\{1, \sigma\}=Z_{2}$. Since $\operatorname{dim}\left(\left(\left(\left(E_{7}\right)^{\kappa, \mu}\right)^{\gamma}\right)_{\dot{F}_{1}\left(h e_{4}\right), \tilde{E}_{1}}\right)=21 \quad($ Lemma 3.10) $=\operatorname{dim}(\mathfrak{s o}(7)), \pi$ is onto. Hence, $\left(\left(\left(E_{7}\right)^{\kappa, \mu}\right)^{\gamma}\right)_{\dot{F}_{1}\left(h e_{4}\right), \tilde{E}_{1}} / Z_{2} \cong S O(7)$. Therefore, $\left(\left(\left(E_{7}\right)^{\kappa, \mu}\right)^{\gamma}\right)_{\dot{F}_{1}\left(h e_{4}\right), \tilde{E}_{1}}$ is isomorphism to $\operatorname{Spin}(7)$ as a double covering group of $S O(7)$.

We shall consider the subgroup $\left(\left(\left(E_{7}\right)^{\kappa, \mu}\right)^{\gamma}\right)_{\dot{F}_{1}\left(h e_{4}\right)}$ of $E_{7}$.

LEMMA 3.14. The Lie algebra $\left(\left(\left(\mathbf{e}_{7}\right)^{\kappa, \mu}\right)^{\gamma}\right)_{\dot{F}_{1}\left(h e_{4}\right)}$ of the group $\left(\left(\left(E_{7}\right)^{\kappa, \mu}\right)^{\gamma}\right)_{\dot{F}_{1}\left(h e_{4}\right)}$ is given by

$$
\begin{aligned}
& \left(\left(\left(\mathrm{e}_{7}\right)^{\kappa, \mu}\right)^{\gamma}\right)_{\dot{F}_{1}\left(h_{4}\right)} \\
= & \left\{\Phi\left(D_{4}+\tilde{A}_{1}(p)+i\left(\begin{array}{ccc}
\varepsilon_{1} & 0 & 0 \\
0 & \varepsilon_{2} & q \\
0 & \bar{q} & \varepsilon_{3}
\end{array}\right),\left(\begin{array}{ccc}
0 & 0 & 0 \\
0 & \alpha_{2} & x \\
0 & \bar{x} & \alpha_{3}
\end{array}\right),-\tau\left(\begin{array}{ccc}
0 & 0 & 0 \\
0 & \alpha_{2} & x \\
0 & \bar{x} & \alpha_{3}
\end{array}\right),-\frac{3}{2} i \varepsilon_{1}\right)\right. \\
& \left.\mid D_{4} \in \mathfrak{s o}(4) \subset \mathfrak{s o}(8), \alpha_{k} \in C, p, q \in \boldsymbol{H}, x \in \boldsymbol{H}^{C}, \varepsilon_{k} \in \boldsymbol{R}, \varepsilon_{1}+\varepsilon_{2}+\varepsilon_{3}=0\right\} .
\end{aligned}
$$


In particular, we have

$$
\operatorname{dim}\left(\left(\left(\left(\mathfrak{e}_{7}\right)^{\kappa, \mu}\right)^{\gamma}\right)_{\dot{F}_{1}\left(h_{4}\right)}\right)=28
$$

Hereafter, any element of the Lie algebra $\left(\left(\left(\mathfrak{e}_{7}\right)^{\kappa, \mu}\right)^{\gamma}\right)_{\dot{F}_{1}\left(h_{4}\right)}$ will be denoted by $\Phi_{8}$.

LeMma 3.15. For $t \in \boldsymbol{R}$, we define a map $\alpha(t)$ of $\mathfrak{P}^{C}$ by

$$
\begin{aligned}
& \alpha(t)(X, Y, \xi, \eta) \\
& =\left(\left(\begin{array}{ccc}
e^{2 i t} \xi_{1} & e^{i t} x_{3} & e^{i t} \bar{x}_{2} \\
e^{i t} \bar{x}_{3} & \xi_{2} & x_{1} \\
e^{i t} x_{2} & \bar{x}_{1} & \xi_{3}
\end{array}\right),\left(\begin{array}{ccc}
e^{-2 i t} \eta_{1} & e^{-i t} y_{3} & e^{-i t} \bar{y}_{2} \\
e^{-i t} \bar{y}_{3} & \eta_{2} & y_{1} \\
e^{-i t} y_{2} & \bar{y}_{1} & \eta_{3}
\end{array}\right), e^{-2 i t} \xi, e^{2 i t} \eta\right) .
\end{aligned}
$$

Then, $\alpha(t) \in\left(\left(\left(E_{7}\right)^{\kappa, \mu}\right)^{\gamma}\right)_{\dot{F}_{1}\left(h e_{4}\right)}$.

ProOF. For $\Phi=\Phi\left(2 i t E_{1} \vee E_{1}, 0,0,-2 i t\right) \in\left(\left(\left(\mathfrak{e}_{7}\right)^{\kappa, \mu}\right)^{\gamma}\right)_{\dot{F}_{1}\left(h_{4}\right)}$ (Lemma 3.14), we have $\alpha(t)=\exp \Phi \in\left(\left(\left(E_{7}\right)^{\kappa, \mu}\right)^{\gamma}\right)_{\dot{F}_{1}\left(h e_{4}\right)}$ by $E_{1} \vee E_{1}=(1 / 3)\left(2 E_{1}-E_{2}-E_{3}\right)^{\sim}$.

We define an 8 dimensional $\boldsymbol{R}$-vector space $V^{8}$ by

$$
\begin{aligned}
V^{8} & =\left\{P \in \mathfrak{P}^{C} \mid \kappa P=P, \mu \tau \lambda P=P, \gamma P=P\right\} \\
& =\left\{P=\left(\left(\begin{array}{ccc}
0 & 0 & 0 \\
0 & \xi & h \\
0 & \bar{h} & -\tau \xi
\end{array}\right),\left(\begin{array}{lll}
\eta & 0 & 0 \\
0 & 0 & 0 \\
0 & 0 & 0
\end{array}\right), 0, \tau \eta\right) \mid \xi, \eta \in C, h \in \boldsymbol{H}\right\}
\end{aligned}
$$

with the norm

$$
(P, P)_{\mu}=\frac{1}{2}(\mu P, \lambda P)=(\tau \xi) \xi+\bar{h} h+(\tau \eta) \eta
$$

Then, $S^{7}=\left\{P \in V^{8} \mid(P, P)_{\mu}=1\right\}$ is a 7 dimensional sphere.

LEMMA 3.16. $\quad\left(\left(\left(E_{7}\right)^{\kappa, \mu}\right)^{\gamma}\right)_{\dot{F}_{1}\left(h e_{4}\right)} / \operatorname{Spin}(7) \simeq S^{7}$. In particular, $\left(\left(\left(E_{7}\right)^{\kappa, \mu}\right)^{\gamma}\right)_{\dot{F}_{1}\left(h e_{4}\right)}$ is connected.

Proof. The group $\left(\left(\left(E_{7}\right)^{\kappa, \mu}\right)^{\gamma}\right)_{\dot{F}_{1}\left(h_{4}\right)}$ acts on $S^{7}$. We shall show that this action is transitive. To show this, it is sufficient to show that any element $P \in S^{7}$ can be transformed to $\left(0, E_{1}, 0,1\right) \in S^{7}$ under the action of $\left(\left(\left(E_{7}\right)^{\kappa, \mu}\right)^{\gamma}\right)_{\dot{F}_{1}\left(h_{4}\right)}$. Now, for a given

$$
P=\left(\left(\begin{array}{ccc}
0 & 0 & 0 \\
0 & \xi & h \\
0 & \bar{h} & -\tau \xi
\end{array}\right),\left(\begin{array}{lll}
\eta & 0 & 0 \\
0 & 0 & 0 \\
0 & 0 & 0
\end{array}\right), 0, \tau \eta\right) \in S^{7}
$$


choose $t \in \boldsymbol{R}$ such that $e^{-2 i t} \eta \in i \boldsymbol{R}$. Operate $\alpha(t)\left(\right.$ Lemma 3.15) $\in\left(\left(\left(E_{7}\right)^{\kappa, \mu}\right)^{\gamma}\right)_{\dot{F}_{1}\left(h e_{4}\right)}$ on $P$. Then,

$$
\alpha(t) P=\left(\left(\begin{array}{ccc}
0 & 0 & 0 \\
0 & \xi & h \\
0 & \bar{h} & -\tau \xi
\end{array}\right),\left(\begin{array}{ccc}
i \eta^{\prime} & 0 & 0 \\
0 & 0 & 0 \\
0 & 0 & 0
\end{array}\right), 0,-i \eta^{\prime}\right)=P_{1} \in S^{6} \subset S^{7}, \quad \eta^{\prime} \in \boldsymbol{R}
$$

Since $\left(\left(\left(E_{7}\right)^{\kappa, \mu}\right)^{\gamma}\right)_{\dot{F}_{1}\left(h h_{4}\right), \tilde{E}_{1}}\left(\subset\left(\left(\left(E_{7}\right)^{\kappa, \mu}\right)^{\gamma}\right)_{\dot{F}_{1}\left(h e_{4}\right)}\right)$ acts transitivity on $S^{6}$ (Lemma 3.12), there exists $\beta \in\left(\left(\left(E_{7}\right)^{\kappa, \mu}\right)^{\gamma}\right)_{\dot{F}_{1}\left(h e_{4}\right), \tilde{E}_{1}}$ such that $\beta P_{1}=\left(0,-i E_{1}, 0, i\right)=P_{2} \in$ $S^{6} \subset S^{7}$. Moreover, operate $\alpha(-\pi / 4)\left(\right.$ Lemma 3.15) on $P_{2}$,

$$
\alpha\left(-\frac{\pi}{4}\right) P_{2}=\left(0, E_{1}, 0,1\right)=\tilde{E}_{1} .
$$

This shows the transitivity. The isotropy subgroup $\left(\left(\left(E_{7}\right)^{\kappa, \mu}\right)^{\gamma}\right)_{\dot{F}_{1}\left(h_{4}\right)}$ at $\tilde{E}_{1}$ is $\left(\left(\left(E_{7}\right)^{\kappa, \mu}\right)^{\gamma}\right)_{\dot{F}_{1}\left(h e_{4}\right), \tilde{E}_{1}}=\operatorname{Spin}(7)$ (Proposition 3.12). Thus, we have the homeomorphism $\left(\left(\left(E_{7}\right)^{\kappa, \mu}\right)^{\gamma}\right)_{\dot{F}_{1}\left(h e_{4}\right)} / \operatorname{Spin}(7) \simeq S^{7}$.

PROPOSITION 3.17. $\quad\left(\left(\left(E_{7}\right)^{\kappa, \mu}\right)^{\gamma}\right)_{\dot{F}_{1}\left(h e_{4}\right)} \cong \operatorname{Spin}(8)$.

Proof. Since $\left(\left(\left(E_{7}\right)^{\kappa, \mu}\right)^{\gamma}\right)_{\dot{F}_{1}\left(h e_{4}\right)}$ is connected (Lemma 3.16), we can define a homormorphism $\pi:\left(\left(\left(E_{7}\right)^{\kappa, \mu}\right)^{\gamma}\right)_{\dot{F}_{1}\left(h e_{4}\right)} \rightarrow S O(8)=S O\left(V^{8}\right)$ by

$$
\pi(\alpha)=\alpha \mid V^{8}
$$

It is not difficult to see that $\operatorname{Ker} \varphi=\{1, \sigma\}=Z_{2}$. Since $\operatorname{dim}\left(\left(\left(\left(E_{7}\right)^{\kappa, \mu}\right)^{\gamma}\right)_{\dot{F}_{1}\left(h e_{4}\right)}\right)=28$ (Lemma 3.14$)=\operatorname{dim}(\mathfrak{s o}(8)), \pi$ is onto. Hence, $\left(\left(\left(E_{7}\right)^{\kappa, \mu}\right)^{\gamma}\right)_{\dot{F}_{1}\left(h_{4}\right)} / Z_{2} \cong S O(8)$. Therefore, $\left(\left(\left(E_{7}\right)^{\kappa, \mu}\right)^{\gamma}\right)_{\dot{F}_{1}\left(h_{4}\right)}$ is isomorphism to $\operatorname{Spin}(8)$ as a double covering group of $S O(8)$.

We shall determine the group structre of $\left(\left(E_{7}\right)^{\kappa, \mu}\right)^{\gamma}$.

Lemma 3.18. The Lie algebra $\left(\left(\mathfrak{e}_{7}\right)^{\kappa, \mu}\right)^{\gamma}$ of the group $\left(\left(E_{7}\right)^{\kappa, \mu}\right)^{\gamma}$ is given by

$$
\begin{aligned}
\left(\left(\mathfrak{e}_{7}\right)^{\kappa, \mu}\right)^{\gamma}= & \\
\Phi & \left(D_{4}+D_{4}^{\prime}+\tilde{A}_{1}(p)+i\left(\begin{array}{ccc}
\varepsilon_{1} & 0 & 0 \\
0 & \varepsilon_{2} & q \\
0 & \bar{q} & \varepsilon_{3}
\end{array}\right),\left(\begin{array}{ccc}
0 & 0 & 0 \\
0 & \alpha_{2} & x \\
0 & \bar{x} & \alpha_{3}
\end{array}\right),\right. \\
& \left.-\tau\left(\begin{array}{ccc}
0 & 0 & 0 \\
0 & \alpha_{2} & x \\
0 & \bar{x} & \alpha_{3}
\end{array}\right),-\frac{3}{2} i \varepsilon_{1}\right) \mid D_{4}, D_{4}^{\prime} \in \mathfrak{s}(4) \subset \mathfrak{s o}(8), \alpha_{k} \in C, p, q \in \boldsymbol{H}, \\
& \left.x \in \boldsymbol{H}^{C}, \varepsilon_{k} \in \boldsymbol{R}, \varepsilon_{1}+\varepsilon_{2}+\varepsilon_{3}=0\right\}
\end{aligned}
$$


In particular, we have

$$
\operatorname{dim}\left(\left(\left(\mathfrak{e}_{7}\right)^{\kappa, \mu}\right)^{\gamma}\right)=34
$$

Proposition 3.19. $\left(\left(E_{7}\right)^{\kappa, \mu}\right)^{\gamma} \cong(\operatorname{Spin}(4) \times \operatorname{Spin}(8)) / Z_{2}, Z_{2}=\{(1,1)$, $(-1,-1)\}$.

ProOF. For $\operatorname{Spin}(4)=S p(1) \times S p(1)=\left(\left(\left(E_{7}\right)^{\kappa, \mu}\right)^{\gamma}\right)_{\dot{F}_{1}(h), \tilde{E}_{1}, \tilde{E}_{-1}, \dot{E}_{23}}$ (Propositions 3.1, 3.3) and $\operatorname{Spin}(8)=\left(\left(\left(E_{7}\right)^{\kappa, \mu}\right)^{\gamma}\right)_{\dot{F}_{1}\left(h_{4}\right)}$ (Proposition 3.17), we define a map $\phi_{1}: \operatorname{Spin}(4) \times \operatorname{Spin}(8) \rightarrow\left(\left(E_{7}\right)^{\kappa, \mu}\right)^{\gamma}$ by

$$
\phi_{1}(\alpha, \beta)=\alpha \beta \text {. }
$$

Then, $\phi_{1}$ is well-defined. For $\Phi_{4} \in \mathfrak{s p i n}(4)\left(\right.$ Lemma 3.2) and $\Phi_{8} \in \mathfrak{s p i n}(8)$ (Lemma 3.14), since $\left[\Phi_{4}, \Phi_{8}\right]=0$, we have $\alpha \beta=\beta \alpha$. Hence, $\phi_{1}$ is a homomorphism. It is not difficult to see that $\operatorname{Ker} \phi_{1}=\{(1,1),(-1,-1)\}=Z_{2}$. Since $\left(\left(E_{7}\right)^{\kappa, \mu}\right)^{\gamma}$ $\left(\cong(\operatorname{Spin}(12))^{\gamma}\right.$. (see [4], [5]) $)$ is connected and $\operatorname{dim}\left(\left(\left(E_{7}\right)^{\kappa, \mu}\right)^{\gamma}\right)=34$ (Lemma $3.18)=6+28=\operatorname{dim}(\mathfrak{s p i n}(4) \oplus \mathfrak{s p i n}(8)), \phi_{1}$ is onto. Thus, we have the required isomorphism $(\operatorname{Spin}(4) \times \operatorname{Spin}(8)) / Z_{2} \cong\left(\left(E_{7}\right)^{\kappa, \mu}\right)^{\gamma}$.

Now, we shall determine the group structure of $\left(E_{7}\right)^{\sigma, \gamma}$.

LEMMA 3.20. The Lie algebra $\left(\mathfrak{e}_{7}\right)^{\sigma, \gamma}$ of the group $\left(E_{7}\right)^{\sigma, \gamma}$ is given by

$$
\begin{aligned}
\left(e_{7}\right)^{\sigma, \gamma}= & \left\{\Phi \left(D_{4}+D_{4}^{\prime}+\tilde{A}_{1}(p)+i\left(\begin{array}{ccc}
\varepsilon_{1} & 0 & 0 \\
0 & \varepsilon_{2} & q \\
0 & \bar{q} & \varepsilon_{3}
\end{array}\right)^{\sim},\left(\begin{array}{ccc}
\alpha_{1} & 0 & 0 \\
0 & \alpha_{2} & x \\
0 & \bar{x} & \alpha_{3}
\end{array}\right),\right.\right. \\
& \left.-\tau\left(\begin{array}{ccc}
\alpha_{1} & 0 & 0 \\
0 & \alpha_{2} & x \\
0 & \bar{x} & \alpha_{3}
\end{array}\right), v\right) \mid D_{4}, D_{4}^{\prime} \in \mathfrak{s o}(4) \subset \mathfrak{s}(8), \alpha_{k} \in C, p, q \in \boldsymbol{H}, x \in \boldsymbol{H}^{C} \\
& \left.\varepsilon_{k} \in \boldsymbol{R}, \varepsilon_{1}+\varepsilon_{2}+\varepsilon_{3}=0, v \in i \boldsymbol{R}\right\}
\end{aligned}
$$

In particular, we have

$$
\operatorname{dim}\left(\left(e_{7}\right)^{\sigma, \gamma}\right)=37
$$

Proposition 3.21. For $A \in S U(2)=\left\{A \in M(2, C) \mid\left(\tau^{t} A\right) A=E\right.$, det $\left.A=1\right\}$, we define C-linear transformations $\phi(A)$ of $\mathfrak{P}^{C}$ by 


$$
\begin{gathered}
\phi(A)\left(\left(\begin{array}{lll}
\xi_{1} & x_{3} & \bar{x}_{2} \\
\bar{x}_{3} & \xi_{2} & x_{1} \\
x_{2} & \bar{x}_{1} & \xi_{3}
\end{array}\right),\left(\begin{array}{lll}
\eta_{1} & y_{3} & \bar{y}_{2} \\
\bar{y}_{3} & \eta_{2} & y_{1} \\
y_{2} & \bar{y}_{1} & \eta_{3}
\end{array}\right), \xi, \eta\right) \\
=\left(\left(\begin{array}{lll}
\xi_{1}^{\prime} & x_{3}^{\prime} & \bar{x}_{2}^{\prime} \\
\bar{x}_{3}^{\prime} & \xi_{2}^{\prime} & x_{1}^{\prime} \\
x_{2}^{\prime} & \bar{x}_{1}^{\prime} & \xi_{3}^{\prime}
\end{array}\right),\left(\begin{array}{lll}
\eta_{1}^{\prime} & y_{3}^{\prime} & \bar{y}_{2}^{\prime} \\
\bar{y}_{3}^{\prime} & \eta_{2}^{\prime} & y_{1}^{\prime} \\
y_{2}^{\prime} & \bar{y}_{1}^{\prime} & \eta_{3}^{\prime}
\end{array}\right), \xi^{\prime}, \eta^{\prime}\right) \\
\left(\begin{array}{c}
\xi_{1}^{\prime} \\
\eta^{\prime}
\end{array}\right)=A\left(\begin{array}{c}
\xi_{1} \\
\eta
\end{array}\right), \quad\left(\begin{array}{l}
\xi^{\prime} \\
\eta_{1}^{\prime}
\end{array}\right)=A\left(\begin{array}{l}
\xi \\
\eta_{1}
\end{array}\right), \quad\left(\begin{array}{l}
\eta_{2}^{\prime} \\
\xi_{3}^{\prime}
\end{array}\right)=A\left(\begin{array}{l}
\eta_{2} \\
\xi_{3}
\end{array}\right) \\
\left(\begin{array}{c}
\eta_{3}^{\prime} \\
\xi_{2}^{\prime}
\end{array}\right)=A\left(\begin{array}{l}
\eta_{3} \\
\xi_{2}
\end{array}\right), \quad\left(\begin{array}{l}
x_{1}^{\prime} \\
y_{1}^{\prime}
\end{array}\right)=(\tau A)\left(\begin{array}{l}
x_{1} \\
y_{1}
\end{array}\right) \\
\left(\begin{array}{c}
x_{2}^{\prime} \\
y_{2}^{\prime}
\end{array}\right)=\left(\begin{array}{l}
x_{2} \\
y_{2}
\end{array}\right), \quad\left(\begin{array}{l}
x_{3}^{\prime} \\
y_{3}^{\prime}
\end{array}\right)=\left(\begin{array}{l}
x_{3} \\
y_{3}
\end{array}\right) .
\end{gathered}
$$

Then, $\phi(A) \in\left(E_{7}\right)^{\sigma, \gamma}$.

Proof. Let $\Phi=\Phi\left(2 v E_{1} \vee E_{1}, a E_{1},-\tau a E_{1}, v\right), a \in C, v \in i \boldsymbol{R}$. Then, $\Phi \in\left(\mathfrak{e}_{7}\right)^{\sigma, \gamma}$ $($ Lemma 3.20)
$\exp \Phi \in\left(E_{7}\right)^{\sigma, \gamma}$. Therefore, for $A=\exp \left(\begin{array}{cc}v & a \\ -\tau a & -v\end{array}\right) \in S U(2)$, we have $\phi(A)=$

Proposition 3.22. $\left(E_{7}\right)^{\sigma} \cong(S U(2) \times \operatorname{Spin}(12)) / Z_{2}, Z_{2}=\{(E, 1),(-E,-\sigma)\}$.

ProOF. The isomorphism is induced by the homomorphism $\varphi_{1}: S U(2) \times$ $\operatorname{Spin}(12) \rightarrow\left(E_{7}\right)^{\sigma}$ by $\varphi_{1}(A, \delta)=\phi(A) \delta$. (In detail, see [4], [5].)

THEOREM 3.23. $\left(E_{7}\right)^{\sigma, \gamma} \cong(S U(2) \times \operatorname{Spin}(4) \times \operatorname{Spin}(8)) /\left(Z_{2} \times Z_{2}\right), Z_{2} \times Z_{2}=$ $\{(E, 1,1),(E, \sigma, \sigma)\} \times\{(E, 1,1),(-E, \gamma,-\sigma \gamma)\}$.

ProOF. For $S U(2)$ (Proposition 3.21), $\operatorname{Spin}(4)=\left(\left(\left(E_{7}\right)^{\kappa, \mu}\right)^{\gamma}\right)_{\dot{F}_{1}(h), \tilde{E}_{1}, \tilde{E}_{-1}, \dot{E}_{23}}$ (Propositions 3.1, 3.3) and $\operatorname{Spin}(8)=\left(\left(\left(E_{7}\right)^{\kappa, \mu}\right)^{\gamma}\right)_{\dot{F}_{1}\left(h e_{4}\right)}$ (Proposition 3.17), we define a map $\varphi: S U(2) \times \operatorname{Spin}(4) \times \operatorname{Spin}(8) \rightarrow\left(E_{7}\right)^{\sigma, \gamma}$ by

$$
\varphi(A, \alpha, \beta)=\phi(A) \alpha \beta \text {. }
$$

Then, $\varphi$ is well-defined. From Propositions 3.19, 3.22, $\varphi$ is a homomorphim. We shall show that $\varphi$ is onto. Let $\rho \in\left(E_{7}\right)^{\sigma, \gamma}$. Since $\left(E_{7}\right)^{\sigma, \gamma} \subset\left(E_{7}\right)^{\sigma}$, there exist $A \in S U(2)$ and $\delta \in \operatorname{Spin}(12)$ such that $\rho=\varphi_{1}(A, \delta)$ (Proposition 3.22). Now, from $\gamma \rho \gamma=\rho$, we have $\phi(A)(\gamma \delta \gamma)=\phi(A) \delta$. Hence, 
Fixed points subgroups by two involutive automorphisms

$$
\left\{\begin{array} { l } 
{ A = A } \\
{ \gamma \delta \gamma = \delta }
\end{array} \text { or } \left\{\begin{array}{l}
A=-A \\
\gamma \delta \gamma=-\sigma \delta
\end{array} .\right.\right.
$$

The latter case is impossible because $A=0$ is false. In the former case, from Proposition 3.19, there exist $\alpha \in \operatorname{Spin}(4)$ and $\beta \in \operatorname{Spin}(8)$ such that $\delta=\phi_{1}(\alpha, \beta)$. Hence, we have

$$
\begin{aligned}
\rho & =\varphi_{1}(A, \delta)=\phi(A) \delta=\phi(A) \phi_{1}(\alpha, \beta) \\
& =\phi(A) \alpha \beta=\varphi(A, \alpha, \beta)
\end{aligned}
$$

It is not difficult to see that

$$
\begin{aligned}
\operatorname{Ker} \varphi & =\{(E, 1,1),(E, \sigma, \sigma),(-E, \gamma,-\sigma \gamma),(-E, \sigma \gamma,-\gamma)\} \\
& =\{(E, 1,1),(E, \sigma, \sigma)\} \times\{(E, 1,1),(-E, \gamma,-\sigma \gamma)\} \\
& =Z_{2} \times Z_{2} .
\end{aligned}
$$

Thus, we have the required isomorphism $(S U(2) \times \operatorname{Spin}(4) \times \operatorname{Spin}(8)) /\left(Z_{2} \times Z_{2}\right) \cong$ $\left(E_{7}\right)^{\sigma, \gamma}$.

\section{References}

[1] T. Miyashita and I. Yokota, Fixed points subgroups $G^{\sigma, \sigma^{\prime}}$ by two involutive automorphisms $\sigma, \sigma^{\prime}$ of compact exceptional Lie group $G=F_{4}, E_{6}$ and $E_{7}$, (2001), preprint.

[2] T. Miyashita and I. Yokota, Fixed points subgroups $G^{\gamma, \gamma^{\prime}}$ by two involutive automorphisms $\gamma, \gamma^{\prime}$ of compact exceptional Lie group $G=G_{2}, F_{4}$ and $E_{6}$ (in Japanese), (2001), preprint.

[ 3 ] I. Yokota, Realizations of involutive automorphisms $\sigma$ and $G^{\sigma}$ of exceptional linear Lie groups $G$, Part I, $G=G_{2}, F_{4}$ and $E_{6}$, Tsukuba J. Math., 4 (1990), 185-223.

[4] I. Yokota, Realizations of involutive automorphisms $\sigma$ and $G^{\sigma}$ of exceptional linear Lie groups $G$, Part II, $G=E_{7}$, Tsukuba J. Math., 14 (1990), 379-404.

[5] I. Yokota, Exceptional simple Lie groups (in Japanese), Gendaisuugakusya, Kyoto, 1992.

Toshikazu Miyashita

Nagano Higashi High School

Nagano, 381-0022, Japan

E-mail: spin15ss16@ybb.ne.jp 